\title{
Measurement of Oxygen Desaturation Is Not Useful for the Detection of Aspiration in Dysphagic Stroke Patients
}

\author{
Thomas Marian Jens Schröder Paul Muhle Inga Claus \\ Stephan Oelenberg Christina Hamacher Tobias Warnecke \\ Sonja Suntrup-Krüger Rainer Dziewas
}

Department of Neurology, University Hospital Münster, Münster, Germany

\author{
Keywords \\ Oxygen desaturation measurement · Aspiration · Dysphagic stroke patients · Fiberoptic \\ endoscopic evaluation of swallowing
}

\section{Abstract}

Background: Dysphagia is one of the most dangerous symptoms of acute stroke. Various screening tools have been suggested for the early detection of this condition. In spite of conflicting results, measurement of oxygen saturation $\left(\mathrm{SpO}_{2}\right)$ during clinical swallowing assessment is still recommended by different national guidelines as a screening tool with a decline in $\mathrm{SpO}_{2} \geq 2 \%$ usually being regarded as a marker of aspiration. This paper assesses the sensitivity of $\mathrm{SpO}_{2}$ measurements for the evaluation of aspiration risk in acute stroke patients. Methods: Fifty acute stroke patients with moderate to severe dysphagia were included in this study. In all patients, fiberoptic endoscopic evaluation of swallowing (FEES) was performed according to a standardised protocol. Blinded to the results of FEES, $\mathrm{SpO}_{2}$ was monitored simultaneously. The degree of desaturation during/after swallows with aspiration was compared to the degree of desaturation during/after swallows without aspiration in a swallow-toswallow analysis of each patient. To minimise potential confounders, every patient served as their control. Results: In each subject, a swallow with and a swallow without aspiration were analysed. Overall, aspiration seen in FEES was related to a minor decline in $\mathrm{SpO}_{2}$ (mean $\mathrm{SpO}_{2}$ without aspiration $95.54 \pm 2.7 \%$ vs. mean $\mathrm{SpO}_{2}$ with aspiration $95.28 \pm 2.7 \%$ ). However, a significant desaturation $\geq 2 \%$ occurred only in 5 patients during/after aspiration. There was no correlation between aspiration/dysphagia severity or the amount of aspirated material and $\mathrm{SpO}_{2}$ levels. Conclusions: According to this study, measurement of oxygen desaturation is not a suitable screening tool for the detection of aspiration in stroke patients. 


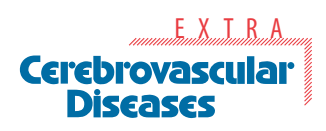

\begin{tabular}{l|l}
\hline Cerebrovasc Dis Extra 2017;7:44-50 \\
\hline DOI: 10.1159/000453083 & $\begin{array}{l}\text { C } 2017 \text { The Author(s). Published by S. Karger AG, Basel } \\
\text { www.karger.com/cee }\end{array}$
\end{tabular}

Marian et al.: Measurement of Oxygen Desaturation Is Not Useful for the Detection of Aspiration in Dysphagic Stroke Patients

\section{Introduction}

Dysphagia is common in stroke and affects up to $80 \%$ of the patients. In the majority of patients, swallowing ability improves within the first 2 weeks after stroke [1-7]. The most feared consequence of dysphagia in acute stroke patients is aspiration, which has been found in $30-51 \%$ of patients, and, in particular, silent aspiration, which has been observed in $8-27 \%$ of patients [3, 8-11]. During the clinical course, dysphagia is associated with an about 3-fold increased risk of mortality mainly due to aspiration pneumonia but also due to malnutrition and dehydration $[1-3,11,12]$.

Pulse oximetry (PO) has been suggested as an option for the detection of aspiration based on the principle that aspiration of food or fluid into the airways leads to bronchospasm or airway obstruction [13], which causes ventilation-perfusion mismatch and a reduction in oxygen saturation $\left(\mathrm{SpO}_{2}\right)$. PO measures $\mathrm{SpO}_{2}$ based on the principle that reduced haemoglobin and oxygenated haemoglobin present different absorption characteristics to infrared light emitted from a finger probe. The method is non-invasive, requires little patient cooperation and is easy to obtain and repeatable.

In spite of these practical advantages of PO, the association of aspiration with oxygen desaturation has been inconclusive. Various studies found no significant association between the reduction in $\mathrm{SpO}_{2}$ and aspiration [14-18], while others reported the contrary, i.e., that PO could be used to detect a high proportion of dysphagic patients with aspiration episodes [1922 ]. A study by Lim et al. [23], for example, attributed a sensitivity of $100 \%$ and a specificity of $71 \%$ to a testing procedure combining fiberoptic endoscopic evaluation of swallowing (FEES) and PO for detecting aspiration. In particular, due to this and similar results, the use of PO for aspiration screening has been included in different guidelines, e.g., in Scotland and Germany [24, 25]. The purpose of the present study, therefore, was to determine the impact of aspiration on $\mathrm{SpO}_{2}$ in a cohort of acute stroke patients using a clean experimental setup enabling a swallow-to-swallow analysis.

\section{Patients and Methods}

\section{Patients}

Fifty acute stroke patients admitted to the stroke unit of the University Hospital Münster during an 8-month period in 2014 were included. Patients with pre-existing dysphagia, other co-morbidities causing dysphagia, a decreased level of consciousness (National Institutes of Health Stroke Scale [NIHSS] subcategory "level of consciousness" $>1$ ) or the need for nasal oxygen supply during FEES were not included in the trial. All examinations were part of our local routine diagnostic workup. Informed consent was obtained from all patients or their next of kin if the patients' communication was impaired. The study was approved by the local ethics committee.

\section{Fiberoptic Endoscopic Evaluation of Swallowing}

Following our in-house guidelines of stroke management, every patient who failed a simple water swallow screening test [26] or showed symptoms predictive of dysphagia, i.e., severe neurological deficit (NIHSS $>10$ points), severe dysarthria/aphasia or facial palsy [27], was further assessed with FEES. Endoscopy was performed in accordance with our protocol for dysphagia assessment in acute stroke, which has been previously developed and validated [28, 29]. Equipment consisted of a 3.1-mm-diameter flexible fiberoptic rhinolaryngoscope (11101RP2, Karl Storz, Tuttlingen, Germany), a light source and camera (rpCam-X, rpSzene ${ }^{\circledR}$, Rehder/Partner, Hamburg, Germany), a colour monitor (WMP-226, 
Wincomm, Taiwan) and a video recorder (AUCC2WV3F, Computar, CBC Group, Japan). All patients were examined by a trained neurologist together with a speech language pathologist. Following the protocol [28], each examined patient was classified according to our 6-point fiberoptic endoscopic dysphagia severity scale (FEDSS) with a score of 1 being best and 6 being worst [29]. In line with guidelines and due to the rapidly changing nature of dysphagia after stroke, FEES was repeated during the first 2 weeks every 3-4 days in patients who were still dysphagic [30].

\section{Measurement of Oxygen Saturation}

$\mathrm{SpO}_{2}$ was measured by a Nellcor ${ }^{\mathrm{TM}}$ fingerclip sending data to a Dräger Infinity ${ }^{\circledR}$ Delta monitor system using the Nellcor ${ }^{\mathrm{TM}}$ OxiMax $^{\mathrm{TM}}$ algorithm. Embedded in the standard FEES workup, $\mathrm{SpO}_{2}$ was monitored by an investigator blinded to the findings of the endoscopist. Each time the patient swallowed, this was announced by the endoscopist, and the second investigator noted $\mathrm{SpO}_{2}$ values during and $2 \mathrm{~min}$ after the swallow. For each swallow, the initial $\mathrm{SpO}_{2}$ level and its lowest level after the swallow were recorded. After completion of FEES, endoscopic findings were matched with the results of PO. For further analysis, only patients were taken into account who exhibited at least 1 liquid swallow with and 1 swallow without aspiration. For each swallow showing aspiration, the previous water swallow without aspiration was chosen as a control. If aspiration occurred during the first swallow (which happened in 15 patients), the next but one swallow without aspiration served as a control. Per patient, 1 swallow with aspiration and a penetration-aspiration scale (PAS) level $\geq 6$ as well as 1 swallow without aspiration were chosen for analysis.

\section{Rating of Aspiration and Dysphagia Severity}

Aspiration severity was rated with the well-established 8-point-interval PAS [31]. For inclusion in this study, swallows with a PAS score of 6-8 were taken into account (PAS 6, ejected aspirated material into the larynx or out of the airway; PAS 7, ejected aspirated material from the trachea despite effort; and PAS 8, made no effort to eject). In addition to the PAS, the degree of aspiration was rated semi-qualitatively according to FEES and depending on bolus size as minor (drops of fluids), massive (nearly complete bolus) and moderate (anything between minor and massive) (Table 1).

\section{Statistical Analyses}

Statistical analyses were carried out with IBM SPSS 23 software (release 23.0). Patient characteristics were summarised using arithmetic means and standard deviations for continuous variables and frequencies and percentages for categorical variables. Continuous and categorical variables were compared using the Kruskal-Wallis test and the $\chi^{2}$ test, respectively. For direct correlation, the Pearson correlation coefficient $(r)$ was used. Sensitivity [true positive/(true positive + false negative)] and specificity [true negative/(true negative + false positive)] were calculated for a relevant desaturation defined as $\mathrm{SpO}_{2}$ decline $\geq 2 \%$, predicting the occurrence of aspiration.

\section{Results}

Fifty patients were eligible for inclusion in this study. During the study period, another 36 patients were screened for eligibility. Twenty-three of these showed no aspiration with PAS $\geq 6$ in any of the liquid swallows; in 8 patients, liquid was not tested because of most severe dysphagia (FEDSS 5 or 6 ); and in 5 FEES, recordings were of poor quality, forestalling study inclusion. The main epidemiological and clinical characteristics of the study cohort are 


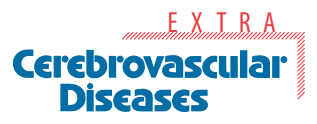

Table 1. Patient characteristics

Table 2. Oxygen saturation

\begin{tabular}{|c|c|}
\hline \multicolumn{2}{|c|}{ Cerebrovasc Dis Extra 2017;7:44-50 } \\
\hline DOI: $10.1159 / 000453083$ & $\begin{array}{l}\text { ( ) } 2017 \text { The Author(s). Published by S. Karger AG, Basel } \\
\text { www.karger.com/cee }\end{array}$ \\
\hline
\end{tabular}

Marian et al.: Measurement of Oxygen Desaturation Is Not Useful for the Detection of Aspiration in Dysphagic Stroke Patients

$\begin{array}{lc}\text { Patients, } n & 50 \\ \text { Sex, } \mathrm{m} / \mathrm{f} & 25 / 25 \\ \text { Mean age } \pm \text { SD, years } & 68.08 \pm 13.62 \\ \text { Ischemic stroke } & 44(88) \\ \text { Haemorrhagic stroke } & 6(12) \\ \text { Mean NIHSS score } \pm \text { SD } & 15.14 \pm 9.82 \\ \text { FEDSS } & \\ \quad \text { Grade 3 } & 24(48) \\ \quad \text { Grade 4 } & 8(16) \\ \quad \text { Grade 5 } & 18(36) \\ \text { PAS } & \\ \quad \text { Grade 6 } & 17(34) \\ \quad \text { Grade 7 } & 18(36) \\ \text { Grade 8 } & 15(30) \\ \text { Aspiration severity } & \\ \quad \text { Minor } & 10(20) \\ \quad \text { Moderate } & 33(66) \\ \quad \text { Severe } & 7(14)\end{array}$

Values are $n(\%)$ unless otherwise indicated. SD, standard deviation; NIHSS, National Institutes of Health Stroke Scale; FEDSS, fiberoptic endoscopic dysphagia severity scale; PAS, penetration-aspiration scale.

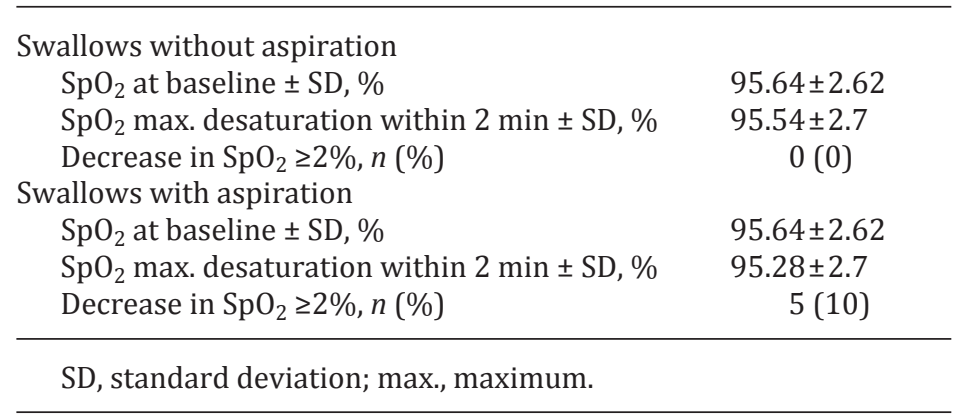

summarised in Table 1. The mean age of the patients was $68 \pm 13.6$ years; $88 \%$ of the strokes were ischemic, and $12 \%$ were haemorrhagic. The mean time from stroke onset to the initial study assessment was $10.26 \pm 14.69$ days. On admission, the patients had a mean NIHSS score of $15.18 \pm 9.72$ points. Thirteen patients underwent intravenous thrombolysis, and 13 patients initially required intubation and artificial ventilation for thrombectomy. All subjects had moderate to severe dysphagia as rated with the FEDSS (Table 1).

Baseline $\mathrm{SpO}_{2}$ was $96 \%$. After swallows with aspiration, a mean $\mathrm{SpO}_{2}$ level of $95.28 \pm$ $2.7 \%$ was measured. Swallows without aspiration showed a mean decline in $\mathrm{SpO}_{2}$ to $95.54 \pm$ $2.7 \%$ (Table 2). Overall, we did not detect a relevant decrease in $\mathrm{SpO}_{2}$ during or after swallowing. In total, only 5 patients (10\%) showed a decrease in $\mathrm{SpO}_{2}$ of $\geq 2 \%$ after swallows with aspiration, whereas this was not detected in any swallows without aspiration (Table 2). These data are related to a corresponding sensitivity of $10 \%$ for $\mathrm{PO}$ (decline in $\mathrm{SpO}_{2} \geq 2 \%$ ) for the detection of aspiration and to a specificity of $100 \%$. In addition, no significant relationship between $\mathrm{SpO}_{2}$ decline and aspiration/dysphagia severity $(k=0.007)$ as well as the amount of aspirated material $(k=0.1101)$ could be detected. 


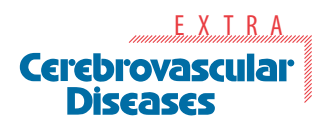

\begin{tabular}{l|l}
\hline Cerebrovasc Dis Extra 2017;7:44-50 \\
\hline DOI: $10.1159 / 000453083$ & $\begin{array}{l}\text { C } 2017 \text { The Author(s). Published by S. Karger AG, Basel } \\
\text { www.karger.com/cee }\end{array}$ \\
\hline
\end{tabular}

Marian et al.: Measurement of Oxygen Desaturation Is Not Useful for the Detection of Aspiration in Dysphagic Stroke Patients

\section{Discussion}

In this study, we investigated whether measurement of $\mathrm{SpO}_{2}$ is helpful for the assessment of aspiration risk in acute stroke patients. The main finding of this study was that there was no statistically significant or even clinically relevant decline in $\mathrm{SpO}_{2}$ after swallows with or without aspiration. In fact, only $10 \%$ of swallows with aspiration (5 out of 50 ) showed a simultaneous decline in $\mathrm{SpO}_{2}$. To the best of our knowledge, this is the first study on this frequently debated topic that used individual patients as their own controls by comparing swallows with and without aspiration in identical patients.

The reasons for this rigorous approach are the heterogeneous results of previous studies and fundamental methodological issues related to the technique of PO. Apart from 2 studies with some relevant problems in study design (Zaidi et al. [19] did not apply instrumental testing, and Lim et al. [23], although using FEES for objective dysphagia assessment, did not study their patients simultaneously with a gold standard method and PO), there are 3 studies with an adequate scientific approach that demonstrated an association between aspiration and oxygen desaturation. Collins and Bakheit [20] in 1997 studied 54 stroke patients by performing PO simultaneously with a videofluoroscopic swallowing study (VFSS). Most importantly, as distinct from later studies, they used larger test boluses during swallowing assessment (150 mL liquids, $3 \mathrm{oz}$ mousse and 1 half of a 2 -inch shortbread biscuit). Their main finding was that $73 \%$ of patients with aspirations were correctly identified by PO, whereas $13 \%$ of patients without aspirations also showed a decline in $\mathrm{SpO}_{2}$ of $\geq 2 \%$ with PO. Interestingly, PO was less sensitive in older patients than in younger ones [20]. A second study by Sherman et al. [21] compared 4 videofluoroscopically defined groups, i.e., (1) aspirators; (2) penetrators without clearing; (3) penetrators with clearing; and (4) patients without penetration or aspiration. Although the authors did not report the sensitivity and specificity of PO for aspiration-related desaturation, they observed that patients with aspiration or penetration without clearing had a significant decline in mean $\mathrm{SpO}_{2}$ compared to the less dysphagic patient groups [21]. Finally, Smith et al. [22] in their study of 53 acute stroke patients reported a sensitivity of $86 \%$ and a specificity of $54 \%$ for $\mathrm{PO}$ (decline in $\mathrm{SpO}_{2} \geq 2 \%$ ) to detect penetration or aspiration as viewed with VFSS. Interestingly, this study featured a substantial number of false-positive patients, giving a positive predictive value of $69 \%$.

In stark contradiction to these observations, 3 equally well-designed studies questioned the validity of PO for determining aspiration risk. Colodny [32] stratified her patient cohort ( $n=181$ ) according to VFSS into "penetrators," "solid aspirators," "liquid aspirators" and "normals." Her main finding was that aspirators had lower $\mathrm{SpO}_{2}$ levels before, during and after swallow testing than non-aspirators. However, no relation was found between $\mathrm{SpO}_{2}$ and aspiration episodes [32]. Adopting a similar design, Leder [15] studied a mixed patient collective $(n=60)$ simultaneously with FEES and PO. His results indicated no significant difference in $\mathrm{SpO}_{2}$ levels based on the patients' aspiration status. Finally, Wang et al. [33] recruited an etiologically heterogeneous group of 60 patients, with stroke being the largest subgroup, but other diseases, such as nasopharyngeal cancer and Parkinsonism, were also present. VFSS was acquired simultaneously with PO. Expanding on the work of Colodny [32] and Leder [15], the authors did not only look for correlations between $\mathrm{SpO}_{2}$ and aspiration status (which again did not exist) but also provided data related to the predictivity of a desaturation episode $\geq 3 \%$ with regard to aspiration on VFSS. The key figures given by the authors were a sensitivity of just $39.1 \%$ and a specificity of $51.7 \%$ for the whole collective and a sensitivity of $58.3 \%$ and a specificity of $66.7 \%$ in the subgroup of stroke patients. In relation to the single episodes of aspiration and desaturation, respectively, Wang et al. [33] reported that of 37 aspirations seen in VFSS, only 11 were combined with a desaturation, and that of 29 desaturation episodes during VFSS, only 12 were found to be in temporal correlation with an aspiration. 
To resolve this puzzle set out by nearly identically designed studies reaching highly divergent conclusions, one needs to take a closer look at other confounding parameters in those investigations. First, the amount of aspirated material might be a crucial factor here. In principle, aspiration of bolus material into the airway might cause direct bronchial obstruction and reflex bronchospasm which in turn lead to ventilation-perfusion mismatch and oxygen desaturation $[13,34]$. Obviously, it seems reasonable to assume that, with an increasing amount of aspirated material, the chance that this physiological reaction may occur will rise. From this perspective, the findings by Collins and Bakheit [20] could well be explained, since this study used, as mentioned above, by far the highest quantity of test boluses of all studies summarised here. Second, one has to take into account that other factors apart from aspiration may have an influence on $\mathrm{SpO}_{2}$ during swallowing, as is evidenced, for example, by the study of Wang et al. [33] describing desaturation episodes that were unrelated to aspiration (see above). Thus, simply changing the body posture during feeding resulted in both significant increase and decrease in $\mathrm{SpO}_{2}$ values in 2 studies [35, 36]. In addition swallow-related desaturation may also be caused by a disturbed coordination of swallowing and breathing and, in particular, by a prolonged deglutition apnoea. In healthy subjects, this episode lasts for $0.25-0.5 \mathrm{~s}$ [37]. In the aged, deglutition apnoea may involve a delay of up to $1 \mathrm{~s}$, and in dysphagic stroke patients, even longer durations of up to $3.4 \mathrm{~s}$ have been observed [38], which may then cause a decline in $\mathrm{SpO}_{2}$. Taken together, oxygen desaturation during feeding is a multi-causal event and not only related to aspiration. To account for this fact, the present study used a design that aimed at minimising potential confounders. Here, individual patients served as their own controls, since single swallows with and without aspiration were analysed from each subject.

In conclusion, $\mathrm{SpO}_{2}$ monitoring does not seem to be helpful to determine aspiration risk in stroke patients. However, since this tool is entirely non-invasive, it might still be used in specific clinical situations. Whereas one cannot deduce anything from an undisturbed pulse oximetry, episodes of desaturation during feeding should be interpreted as unspecific "alarm" signals and should induce further thorough assessments.

\section{Disclosure Statement}

Thomas Marian, Jens Schröder, Paul Muhle, Inga Claus, Stephan Oelenberg, Christina Hamacher and Sonja Suntrup-Krüger declare no conflicts of interest. Tobias Warnecke has received lecture fees from Abbvie, Teva, Bayer and UCB, worked as a consultant for Abbvie and UCB and received a research grant from Deutsche Parkinson Vereinigung e.V. (dPV). Rainer Dziewas received lecture fees from Nutricia, Fresenius Kabi, Boehringer Ingelheim, Bayer, Pfizer and Daiichi Sankyo, worked as a Consultant for Nestle, Daichii Sankyo and InfectoPharm and is a member of the clinical advisory board of Phagenesis.

\section{References}

1 Gordon C, Hewer RL, Wade DT: Dysphagia in acute stroke. Br Med J Clin Res Ed 1987;295:411-414.

-2 Barer DH: The natural history and functional consequences of dysphagia after hemispheric stroke. J Neurol Neurosurg Psychiatry 1989;52:236-241.

-3 Smithard DG, O'Neill PA, Parks C, Morris J: Complications and outcome after acute stroke. Does dysphagia matter? Stroke J Cereb Circ 1996;27:1200-1204.

4 Smithard DG, Smeeton NC, Wolfe CDA: Long-term outcome after stroke: does dysphagia matter? Age Ageing 2007;36:90-94.

5 Nilsson H, Ekberg 0, Olsson R, Hindfelt B: Dysphagia in stroke: a prospective study of quantitative aspects of swallowing in dysphagic patients. Dysphagia 1998;13:32-38.

-6 Martino R, Foley N, Bhogal S, Diamant N, Speechley M, Teasell R: Dysphagia after stroke: incidence, diagnosis, and pulmonary complications. Stroke J Cereb Circ 2005;36:2756-2763. 
-7 Baroni AFFB, Fábio SRC, Dantas RO: Risk factors for swallowing dysfunction in stroke patients. Arq Gastroenterol 2012;49:118-124.

-8 Horner J, Massey EW, Riski JE, Lathrop DL, Chase KN: Aspiration following stroke: clinical correlates and outcome. Neurology 1988;38:1359-1362.

-9 Splaingard ML, Hutchins B, Sulton LD, Chaudhuri G: Aspiration in rehabilitation patients: videofluoroscopy vs bedside clinical assessment. Arch Phys Med Rehabil 1988;69:637-640.

10 Kidd D, Lawson J, Nesbitt R, MacMahon J: The natural history and clinical consequences of aspiration in acute stroke. QJM 1995;88:409-413.

11 Mann G, Hankey GJ, Cameron D: Swallowing function after stroke prognosis and prognostic factors at 6 months. Stroke 1999;30:744-748.

12 Katzan IL, Cebul RD, Husak SH, Dawson NV, Baker DW: The effect of pneumonia on mortality among patients hospitalized for acute stroke. Neurology 2003;60:620-625.

-13 Rodriguez-Roisin R, Ferrer A, Navajas D, Agusti AG, Wagner PD, Roca J: Ventilation-perfusion mismatch after methacholine challenge in patients with mild bronchial asthma. Am Rev Respir Dis 1991;144:88-94.

14 Sellars C, Dunnet C, Carter R: A preliminary comparison of videofluoroscopy of swallow and pulse oximetry in the identification of aspiration in dysphagic patients. Dysphagia 1998;13:82-86.

$\rightarrow 15$ Leder SB: Use of arterial oxygen saturation, heart rate, and blood pressure as indirect objective physiologic markers to predict aspiration. Dysphagia 2000;15:201-205.

$\checkmark 16$ Colodny N: Interjudge and intrajudge reliabilities in fiberoptic endoscopic evaluation of swallowing (FEES) using the penetration-aspiration scale: a replication study. Dysphagia 2002;17:308-315.

17 Wang Y, Lim LL-Y, Heller RF, Fisher J, Levi CR: A prediction model of 1-year mortality for acute ischemic stroke patients. Arch Phys Med Rehabil 2003;84:1006-1011.

18 Ramsey DJC, Smithard DG, Kalra L: Can pulse oximetry or a bedside swallowing assessment be used to detect aspiration after stroke? Stroke 2006;37:2984-2988.

19 Zaidi NH, Smith HA, King SC, Park C, O’Neill PA, Connolly MJ: Oxygen desaturation on swallowing as a potential marker of aspiration in acute stroke. Age Ageing 1995;24:267-270.

20 Collins MJ, Bakheit AM: Does pulse oximetry reliably detect aspiration in dysphagic stroke patients? Stroke J Cereb Circ 1997;28:1773-1775.

-21 Sherman B, Nisenboum JM, Jesberger BL, Morrow CA, Jesberger JA: Assessment of dysphagia with the use of pulse oximetry. Dysphagia 1999;14:152-156.

22 Smith HA, Lee SH, O'Neill PA, Connolly MJ: The combination of bedside swallowing assessment and oxygen saturation monitoring of swallowing in acute stroke: a safe and humane screening tool. Age Ageing 2000;29:495-499.

23 Lim SH, Lieu PK, Phua SY, Seshadri R, Venketasubramanian N, Lee SH, et al: Accuracy of bedside clinical methods compared with fiberoptic endoscopic examination of swallowing (FEES) in determining the risk of aspiration in acute stroke patients. Dysphagia 2001;16:1-6.

24 Deutsche Gesellschaft für Neurologie: Neurogene Dysphagien. Leitlinien der DGN 2008. http://www.dgn.org/ images/red_leitlinien/LL_2008/archiv/ll08kap_098.pdf (accessed February 2, 2016).

25 SIGN Guideline 119: Management of Patients with Stroke: Identification and Management of Dysphagia. http://www.sign.ac.uk/guidelines/fulltext/119/contents.html (accessed February 2, 2016).

-26 Prosiegel M, Riecker A, Weinert M, Dziewas R, Lindner-Pfleghar B, Stanschus S, et al: Management of dysphagic patients with acute stroke (in German). Nervenarzt 2012;83:1590-1599.

-27 Falsetti P, Acciai C, Palilla R, Bosi M, Carpinteri F, Zingarelli A, et al: Oropharyngeal dysphagia after stroke: incidence, diagnosis, and clinical predictors in patients admitted to a neurorehabilitation unit. J Stroke Cerebrovasc Dis 2009;18:329-335.

-28 Dziewas R, Warnecke T, Olenberg S, Teismann I, Zimmermann J, Kramer C, et al: Towards a basic endoscopic assessment of swallowing in acute stroke development and evaluation of a simple dysphagia score. Cerebrovasc Dis 2008;26:41-47.

29 Warnecke T, Ritter MA, Kroger B, Oelenberg S, Teismann I, Heuschmann PU, et al: Fiberoptic endoscopic dysphagia severity scale predicts outcome after acute stroke. Cerebrovasc Dis 2009;28:283-289.

-30 Wirth R, Smoliner C, Jäger M, Warnecke T, Leischker AH, Dziewas R: Guideline clinical nutrition in patients with stroke. Exp Transl Stroke Med 2013;5:14.

31 Rosenbek JC, Robbins JA, Roecker EB, Coyle JL, Wood JL: A penetration-aspiration scale. Dysphagia 1996;11: 93-98.

-32 Colodny N: Comparison of dysphagics and nondysphagics on pulse oximetry during oral feeding. Dysphagia 2000;15:68-73.

-33 Wang T-G, Chang Y-C, Chen S-Y, Hsiao T-Y: Pulse oximetry does not reliably detect aspiration on videofluoroscopic swallowing study. Arch Phys Med Rehabil 2005;86:730-734.

34 Colebatch HJH, Halmagyi DFJ: Reflex airway reaction to fluid aspiration. J Appl Physiol 1962;17:787-794.

-35 Tamura F, Shishikura J, Mukai Y, Kaneko Y: Arterial oxygen saturation in severely disabled people: effect of oral feeding in the sitting position. Dysphagia 1999;14:204-211.

-36 Rogers B, Msall M, Shucard D: Hypoxemia during oral feedings in adults with dysphagia and severe neurological disabilities. Dysphagia 1993;8:43-48.

37 Clark GA: Deglutition apnoea. J Physiol (Lond) 1920;54:59.

-38 Selley WG, Flack FC, Ellis RE, Brooks WA: Respiratory patterns associated with swallowing: part 2. Neurologically impaired dysphagic patients. Age Ageing 1989;18:173-176. 